\title{
EDITORIAL
}

\section{Algunos aspectos del artículo científico en la Revista Peruana de Biología}

\author{
Some aspects of scientific article in Revista Peruana de Biología
}

\section{Leonardo Romero}

Facultad de Ciencias Biológicas, Universidad Nacional Mayor de San Marcos. Ciudad Universitaria de San Marcos, Facultad de Ciencias Biológicas, Calle Germán Amézaga Nº 375, Lima 1 - Perú.

Iromeroc@unmsm.edu.pe

\section{Resumen}

En este trabajo se presentan algunas de las características de los artículos de la Revista Peruana de Biología. Se precisan algunos aspectos del proceso de revisiones, el tipo de artículo conocido como data paper, el problema en la falta de erudición en los trabajos presentados, la importancia de la reproducibilidad y la autoría.

Palabras clave: revisión por pares; artículo científico; reproducibilidad; autoría; erudición.

\section{Abstract}

This paper presents some characteristics of the articles of Revista Peruana de Biologia. Aspects of the peer review process, about article type: the data paper, the problem on lack of scholarship in the submitted manuscripts, the importance of reproducibility and authorship.

Keywords: peer review; scientific article; reproducibility; authorship; erudition.

\section{Calidad de un artículo científico}

\section{“¿su revista es ISI?” [un posible autor]}

Podríamos decir que el concepto de calidad del artículo científico depende de la persona que hace la pregunta. Por ejemplo, calidad para un lector inmediato, dependerá del contendido y que tan fácil accedió al artículo; para los autores la calidad estará en relación con el reconocimiento y las citas logradas; para la casa editora, el artículo debe darle prestigio, asiduos autores y lectores; para el bibliotecólogo, debe estar normalizado, ser interoperable para que pueda entrar fácilmente en sus sistemas; para otros académicos la calidad puede depender de cuan preparado está el artículo para sobrevivir en el ciberespacio; para otros, si el artículo puede afrontar escenarios futuros que la tecnología y la sociedad le impondrán, tal como los paradigmas del acceso abierto, los formatos dinámicos, el Altmetrics, la autoría colaborativa, entre muchos conceptos más [1], pero, debemos advertir que de estos últimos conceptos, algunos ya están puestos en práctica o germinando en la Revista Peruana de Biología. Las siguientes líneas son comentarios de ellos, mostrando algunos vínculos relevantes, que presentan someramente su escenario y muestran que la calidad del artículo científico tiene más dimensiones de las que nos involucra directa e inmediatamente. También, espero contribuir a erradicar del imaginario científico la perversa relación entre calidad del artículo y el termino ISI, (Institute for Scientific Information y que en la actualidad no existe) mostrando que nos enfrentamos a un proceso muy dinámico, complejo y globalizado del desarrollo científico y de su comunicación.

\section{Citación:}

Romero L. 2015. Algunos aspectos del artículo científico en la Revista Peruana de Biología. Revista peruana de biología 22(3): 269 - 274 (Diciembre 2015). doi: http://dx.doi.org/10.15381/rpb.v22i3.11431

$\begin{array}{ll}\text { Presentado: } & 12 / 12 / 2015 \\ \text { Aceptado: } & 24 / 12 / 2015\end{array}$

Publicado online: $30 / 12$ 


\section{Características del artículo científico en la RPB}

"Usted no quiere publicar mi trabajo porque lo considera de mala calidad" [un autor]

Para definir el rumbo de una revista científica tenemos que pasar ineludiblemente por reflexionar sobre las características de los artículos que se publicarán. Considero que calificar un manuscrito con el término de calidad en algún grado no es apropiado, porque este trabajo será sometido a un proceso de revisión y edición, que al final coadyuvaran para que presente las características que la revista exige ya sea en presentación, estilo y contenido; por lo tanto, el concepto de calidad del artículo estará en relación a las características que exige la revista. Y aquí, debemos tomar en cuenta que las revistas varían mucho en las características y los detalles que exigen, esto ocurre en parte relacionado a diferentes aspectos como: la comunidad científica/ académica/profesional que la sustenta, la casa editora, el propósito de la revista, su economía, los editores, y otros tantos más.

Por los motivos expuestos, muchos libros sobre redacción científica $[2,3,4,5,6,7]$ incluyen capítulos para orientar a los investigadores sobre la elección de la revista apropiada y como preparar sus trabajos (de acuerdo al tipo de investigación, la comunidad, usos y estilos, y condiciones técnicas - por ejemplo el envío del manuscrito en Latex, entre otros aspectos). Todos estos libros coinciden en señalar que si no se conoce bien a la revista podríamos tener contratiempos, por ejemplo por diversos motivos el trabajo seria rechazado, y en el peor de los casos (sí, en el peor de los casos) si es aceptado podría permanecer desconocido porque ha quedado oculto en una publicación que sus pares científicos no consultarán. Por lo tanto, un manuscrito siempre es perfectible, y su contenido si es una información valiosa y relevante debe ser publicado.

Aunque la Revista Peruana de Biología (RPB) enuncia varias características que deben tener los trabajos presentados, y que son tomados en cuenta en una inicial evaluación, posteriormente se lleva a cabo el proceso de revisiones (peer review). Este proceso de revisiones es encargado a un Editor Asociado, o a un Editor Académico, que es un académico invitado a llevar el proceso de revisiones. Los editores buscan uno o más revisores para tener opiniones expertas, y luego envían un informe al Editor Jefe. Existen varios modelos de revisiones, y el que utiliza la RPB es el simple (Single blind), que a su vez es el más común $\left.{ }^{8}\right]$. Sin embargo, los modelos de revisión van cambiando y adecuándose a diversos aspectos tecnológicos como la INTERNET [ $\left.{ }^{9}\right]$ y requerimientos de transparencia editorial. Llevados por esta transparencia, incluso algunas revista han puesto en práctica la publicación de los comentarios o informes de los revisores [10, $\left.{ }^{11}\right]$ los que además suelen enriquecer el artículo.

El juicio de los editores y revisores está basado en experiencia y conocimiento lo que le permite proporcionar valiosos comentarios que permiten identificar los errores y corregirlos, detectar malos procedimientos y fraudes. Podríamos ensayar un esquema que nos muestre sintéticamente los aspectos que son más importantes (Fig. 1), obviando posibles fraudes y malos comportamientos (p.e.: plagios). El balance apropiado de esos elementos conduciría a la publicación del artículo, una tendencia hacia la derecha nos proporcionaría un artículo más "exitoso".

\section{El data paper y la usabilidad de la información}

“... mi trabajo no es un dato..." [un autor]

Los artículos publicados en la RPB según la UNESCO [12] pertenecen a la categoría de memorias originales o publicaciones primarias. Dentro de esta categoría tenemos varios tipos como los Trabajos originales, Notas científicas, Revisiones y Comentarios, que se distinguen entre ellos básicamente por la longitud del texto y por el tipo de comunicación. Estos tipos de artículos son bastante comunes en las revistas de la misma temática que la RPB, sin embargo, la intensidad con que se desarrollan las investigaciones, incrementado la producción de artículos científicos (y revistas) a nivel mundial, y la suma de la INTERNET y su tecnología como un medio de difusión a la actividad de la publicación científica han conllevado a otros tipos de artículos. En muchos casos ciertos tipos de artículos han perdido su sentido como comunicación o han encontrado un sustituto dentro de otros medios, por ejemplo muchos tipos de notas taxonómicas ante el uso de bases de datos. Pero también han surgido nuevas necesidades, unas relacionadas con el acceso a la información que

características del artículo científico

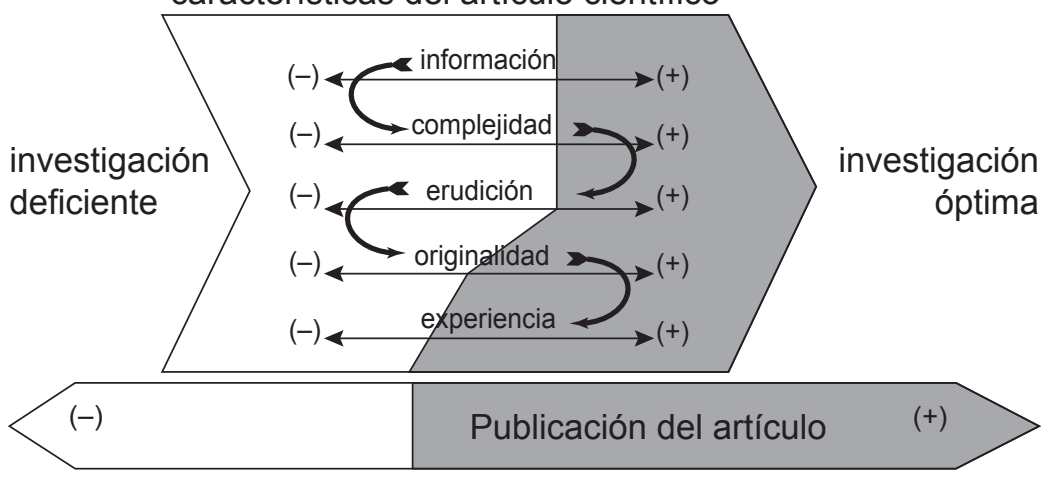

Figura 1. Este esquema señala los aspectos que pueden dar relevancia al trabajo, pero debe recalcarse que es un modelo conceptual. Un manuscrito debe cumplir ciertas características, de ellas algunas como: la información relevante, el grado de complejidad y la erudición son importantes para que un artículo tenga más probabilidades de ser aceptado y publicado. Mientras que la originalidad/creatividad y la experiencia del investigador estructurando artículos son elementos bastante variables, dependen de varias circunstancias y se permite tolerancia, pero los tres primeros son más estrictos. 
fue financiada con recursos del estado (Open Accces, Open Data), otras con el interés de las comunidades científicas de explorar la información, y también con la necesidad de acelerar el paso de la información a la comunidad $\left[{ }^{13}\right]$.

Revisando artículos científicos, además de los tipos de artículos convencionales, podemos encontrar trabajos con abundante información, algo así como bases datos. En el otro extremo observamos artículos que describen un hallazgo, un evento, un caso clínico, el reporte de una especie en un lugar inesperado, el estado de contaminación de una bahía; algunos acompańados de cientos de necesarios análisis para corroborar el hallazgo, y otros con una simple foto como ilustración. Ambos tipos de artículos son interesantes para la comunidad científica, son útiles y deben ser publicados con las normas y condiciones adecuadas para que se respeten los derechos de autoría, otras consideraciones éticas y puedan fácilmente ser utilizados por la comunidad científica.

La publicación de archivos de datos, en unos casos como apéndices, en otros como archivos debidamente registrados y en formatos que puedan ser transferidos a maquinas (p.e.: hojas de cálculo, XML, JSON) no son de reciente uso y algunas editoras, como el BioMed Central solicitan poner a disposición de los lectores los datos en repositorios abiertos [14]. Desde el año 2000 en los archivos de las publicaciones de la Sociedad Ecológica Americana (Ecological Society of America, ESA) [ $\left.{ }^{15}\right]$ se ha venido publicando información extra, conformada principalmente por archivos de los datos (apéndices, suplementos y data papers) utilizados en los artículos de sus revistas. Estos archivos son utilizados en posteriores análisis por la comunidad científica, y también cubren un aspecto de trazabilidad. Desde hace unos años se viene poniendo en práctica la publicación de un nuevo tipo de artículo: el data paper, en nuestro caso biodiversity data paper.

Un data paper es un artículo en una revista, cuyo objetivo principal es describir datos; no tiene hipótesis, ni discute los datos, como un artículo de investigación convencional. Sus objetivos son tres: proporcionar una publicación citable que proporcione los créditos académicos a los autores [editores] así como a las instituciones correspondientes; proporcionar los datos en un formato estructurado de datos amigables [leíbles] para humanos y maquinas (Readable by humans and machines); y hacer visible los datos a la comunidad académica para su uso $\left[{ }^{16}\right]$. Entre las características de estos artículos están la de ser publicaciones digitales, con metadatos definidos como el GBIF Metadata Profile (GMP) $\left.{ }^{17}\right]$, basado en el Ecological Metadata Language (EML) [ $\left.{ }^{18}\right]$, o el Darwin Core (DwC) $\left.{ }^{19}\right]$. Algunas revistas como la Earth System Science Data (ESSD) $\left[{ }^{20}\right]$ y la Scientific Data $\left.{ }^{21}\right]$ se dedican exclusivamente a publicar este tipo de artículos, mientras otras como ZooKeys[22] y Biodiversity Data Journal [23] han implementado este tipo de artículos de datos.

Entre las finalidades de publicar estos archivos de datos en la categoría de artículos es la mantener el reconocimiento de la autoría, pero también de combatir lo que se conoce como el Dark Data [24] que sería la información escondida en los archivos de laboratorios, repositorios institucionales, así como en los apéndices y tablas de los artículos de revistas y a los que no se pueden tener acceso o no existe un medio adecuado de tener acceso a esa información, toda esa información conformaría lo que se ha venido en denominar la Long Tail of Science.

\section{El trabajo insipiente y la erudición del investigador}

"El trabajo usa citas inapropiadas e irrelevantes" [un revisor]

Un trabajo que analiza información, variables, factores, etc. debe recurrir a conceptos y análisis estadísticos, el trabajo se enfrenta a mayor complejidad, con hipótesis elaboradas y a su vez más informativas. Las fallas frecuentes en estos trabajos es la superficialidad con que se enfrenta la argumentación y la explicación de los conceptos y sus análisis.

La argumentación en un artículo científico está ligada a la intertextualidad $\left[{ }^{25}\right]$, aspecto característico en el cual nuestro texto incluye citas a otros textos enriqueciendo el contenido. Entre los problemas de argumentación, tenemos el no recurrir a las referencias fuente $[26,27,28]$ optando por utilizar citas inapropiadas e irrelevantes que no sustentan las afirmaciones del texto. Desde la aparición de la INTERNET y su profunda penetración en la vida académica, haciendo visible y asequible todo tipo de información, no utilizar las referencias fuentes no puede ser tolerado.

Sin embargo, no es solo la insipiencia y la superficialidad, sino la falta experticia en el análisis los datos, así como la ausencia en el uso de conceptos y procedimientos estadísticos, lo que conlleva a la incapacidad de demostrar convincentemente la hipótesis con la evidencia conseguida. El diseño y el análisis estadístico constituyen una parte muy importante del trabajo científico, y nos dice del nivel de conocimiento al que se está llegando. Pasamos de una investigación descriptiva o exploratoria donde la información es el núcleo del artículo, a una investigación explicativa o correlacional, donde a la información se suma el análisis, conceptos, teorías e hipótesis.

El artículo científico es una comunicación, por lo tanto se sustenta en una idea central sobre un problema delimitado y un objetivo bien definido, generalmente se suma una sustanciosa hipótesis que nos lleve a decir que "efectivamente el artículo aporta al conocimiento”. Esta idea es el centro de la originalidad del trabajo, es decir el componente creativo, novedoso, opuesto a la copia, plagio o derivación de alguna otra obra. Esta idea se fundamenta en el conocimiento y la experiencia del investigador y se pueden apreciar en la sección de la introducción del artículo, que es una sección donde la academia, el dominio de la literatura y la retórica son esenciales, aquí el lector debe valorar la información que se brinda, convencerse de la importancia del trabajo, su justificación, debe encontrar el sentido y el contexto al objetivo del trabajo. El descuido de esta sección del artículo, mostrando ideas inconexas, adornadas de citas fuera de lugar, sin lograr la valorización del artículo, causa un desmedro y es un motivo para no aceptarlo o calificar la obra como de "mala calidad".

\section{Reproducibilidad del trabajo}

\section{“¿Qué es un falso positivo?”[un autor]}

"Las bajas tasas de reproducibilidad dentro de la investigación en ciencias biológicas socavan la producción de conocimiento acumulado y contribuyen a los retrasos y los incrementos en los costos de desarrollo de nuevos fármacos. Un análisis de publicaciones biomédicas indica que la investigación preclínica irreproducible supera el $50 \%$, lo que resulta en aproximadamente US \$ 28 mil millones por ańo de gastos solamente en los Estados Unidos" [traducción libre] [ $\left.{ }^{29}\right]$. 
Tabla 1. La siguiente tabla fue presentada por Allen et al. (2014) y elaborada basada en una encuesta realizada en el año 2013, a 1200 autores para correspondencia, de artículos publicados en PLOS journals, Nature Publishing Group journals, Elsevier journals, Science y eLife. La encuesta preguntaba sobre las contribuciones que habían tenido los coautores tomando en cuenta la categoría taxonómica de rol. La respuesta fue de 230 autores, de ellos más del $85 \%$ consideraron que el categoría taxonómica fue fácil de usar y que cubría todos los roles o actividades de los contribuidores. Esta encuesta fue una investigación exploratoria piloto, donde los encuestados fueron de las áreas biomédicas y ciencias de la vida; y fue motivada por International Workshop on Contributorship and Scholarly Attribution (mayo 2012) [http://projects.iq.harvard. edu/files/attribution_workshop/files/iwcsa_report_final_18sept12.pdf]. Esta iniciativa surgió como una respuesta al notorio incremento de los autores en los trabajos de investigación, y la complejidad que adquieren los artículos, con las diferentes organizaciones participantes, y las entidades que las financian. Ante la clásica concepción de autoría, y el no considerar los roles adecuados, surge esta propuesta que está dirigida a dar transparencia en una diversidad de tipos de contribución a la obra y que deben ser reconocidos. [revisar el Blog Scielo en Perspectiva, http://blog.scielo.org/es/2014/07/17/los-creditosdel-autor-autor-de-que/]

Categoría taxonómica de rol

Concepción del estudio

Metodología

Computación

Análisis formal

Investigación: realización de experimentos

Investigación: recolección de datos/

evidencias, muestreos

Recursos

Curatoria de datos

Preparación del manuscrito: escribir el primer borrador

Preparación del manuscrito: revisión critica, comentarios

Preparación del manuscrito: ilustraciones/ presentación de datos

Supervisión

Administrador del proyecto

Consecución de fondos

\section{Descripción del rol}

Proporciona la ideas central, la pregunta de investigación, establece la hipótesis

Desarrolla o diseña la metodología, creación de modelos.

Desarrollo de software, programación, análisis computacional, creación de código (script) y de algoritmos.

Aplicación de estadísticas, técnicas matemáticas otros análisis formales.

Conduce el proceso de investigación, diseño de los experimentos.

Conduce el proceso de investigación, diseño de la recolección de datos/ evidencias, muestreos.

Provee los materiales de estudio, reactivos, muestras de laboratorio, animales, instrumental y otras herramientas para el análisis.

Encargado de la introducción, clasificación de datos y metadatos producidos y organizar los datos para el uso inicial y posterior reutilización.

Preparación, creación y presentación del manuscrito inicial.

Preparación del manuscrito, revisión crítica, comentarios.

Preparación, creación de las ilustraciones del manuscrito (tablas y figuras)

Responsable de la supervisión de la investigación, organización y administración del proyecto, investigador principal o líder y tomador de decisiones.

Coordinación o administración de las actividades de investigación que conducen a la publicación.

Consecución de soporte financiero y logístico para que el proyecto llegue a publicación.

(*) Allen, Liz, Jo Scott, Amy Brand, Marjorie Hlava, y Micah Altman. 2014. «Publishing: Credit where credit is due». Nature 508 (7496): 312-13. doi:10.1038/508312a. [traducción libre]

El párrafo anterior hace referencia a diferentes trabajos publicados y puestos a prueba, por ejemplo en experimentos preclínicos de medicina $\left[{ }^{30}\right]$, sobre el cáncer $\left[{ }^{31}\right]$, y sobre el efecto de drogas y fármacos $\left[{ }^{32}\right]$, donde todos encontraron un alto porcentaje de estudios irreproducibles y muy pocos eran completamente reproducibles. También se observó esto en investigaciones psicológicas donde se encontró que casi todas eran irreproducibles [33], así como en el campo de la biología molecular y que causaron varias iniciativas para estandarizar las metodologías utilizadas [34]. Todos estos artículos que no podían volver a repetirse, por falta de información en al artículo, fueron calificados como malos comportamientos y con sospechas de fraudulentos, y llevaron a revistas como Nature a tomar medidas $\left.{ }^{35}\right]$ para contrarrestar estos casos y utilizar Reporting Checklist para los manuscritos $\left.{ }^{36}\right]$. Sin embargo, los problemas son más complicados porque esta situación es señalada como uno de los causantes del incremento de retracciones en las revistas científicas $[37,38,39]$.

Una de las características de las publicaciones científicas es incluir la información suficiente y detallada para lograr la reproducibilidad de los experimentos, esto permite poner a prueba los métodos y la información, así como también se considera una forma de transferencia de conocimiento y es un aspecto tratado a nivel epistemológico [ $\left.{ }^{40,} 41\right]$. Por lo tanto, la descripción correcta y 
debidamente detallada de los materiales y métodos debe tomarse en cuenta para que permita la reproducibilidad del experimento o la observación. Son buenas prácticas, poner énfasis en describir los procedimientos que validan el diseño experimental o de colecta y por qué se realiza de esa manera, analizar las fuentes de error (aleatorio y sistemático), ilustrar con diagramas y si hubiera antecedentes deben mencionarse. También debe tenerse cuidado en la explicación de cada procedimiento estadístico utilizado. Los autores deben tener en consideración que sus lectores evaluaran la calidad de los datos a utilizarse.

\section{La autoría como indicador de un buen trabajo de investigación}

\section{¿Por qué el autor para correspondencia? [un autor]}

Desde hace mucho tiempo, el tema de la autoría del artículo científico ha sido tratado como un problema ético. El International Committee of Medical Journal Editors (ICMJE) considera cuatro condiciones para ser autor $\left[{ }^{42}\right]$, y resalta aquella donde se atribuye las responsabilidades de la obra publicada a todos por igual. Esto lleva a declaraciones explicitas de su condición de autores asumiendo sus responsabilidades.

Pero además, una propuesta que va siendo adoptada por las revistas científicas es considerar el rol especifico que jugaron los autores en la elaboración del artículo [43,44]. Es una buena práctica para el reconocimiento de las actividades de investigación y está en relación a investigaciones realizadas en escenarios formales de colaboración y cooperación $\left[{ }^{45}\right]$. Estos conceptos han llevado a la propuesta de una taxonomía de roles de autoría $\left[{ }^{46}\right]$ (Tabla 1). Algunos comentarios al respecto han comparado la mención de los roles como el reparto de los premios Oscar de la Academia de Cine Norteamericana, y también existen propuestas de tener en el futuro una base de datos de estos roles. Esta taxonomía no excluye las condiciones consideradas por el ICMJE.

Algo que los autores deben tomar muy en serio es que el trabajo presentado es su obra. Todos tienen los mismos créditos y derechos, en la RPB los derechos de Copyrigh recaen sobre los autores con una licencia de Creative Commons ReconocimientoCompartirIgual 4.0 Internacional. Pero también tienen las mismas responsabilidades por igual. Sin embargo, por motivos prácticos se nombra al autor que presenta la obra como el autor para correspondencia y esto es señalado en el artículo, pues es con él con quien el Editor Jefe coordina las correcciones, la aprobación final del trabajo y el consentimiento para la publicación.

\section{Referencias}

1 Bartling, Sönke, y Sascha Friesike, eds. 2014. Opening Science. Cham: Springer International Publishing. http://link.springer.com/10.1007/978-3-319-00026-8.

2 Day, Robert A. 2005. Como escribir y publicar trabajos cientificos. Traducción de la 5 th edición. Washington, D.C: Organización Panamericana de la Salud (OPS).

3 Nair, P.K. Ramachandran, y Vimala D. Nair. 2014. Scientific Writing and Communication in Agriculture and Natural Resources. Cham: Springer International Publishing. http://link.springer.com/10.1007/978-3-319-03101-9.

4 Peat, Jennifer K, Elizabeth Elliott, Louise Baur, y Victoria Keena. 2002. Scientific Writing Easy When You Know How. London: John Wiley \& Sons, Inc.

5 Youdeowei, Anthony, Paul Stapleton, y Rodger Obubo, eds. 2012. Scientific Writing for Agricultural Research Scientists: A Training Resource Manual. Wageningen, The Netherlands: The Technical Centre for Agricultural and Rural Cooperation (CTA).
6 Cargill, Margaret, y Patrick O'Connor. 2013. Writing Scientific Research Articles: Strategy and Steps. 2 edition. WileyBlackwell.

7 Belcher, Wendy Laura. 2012. Cómo escribir un artículo académico en 12 semanas: guía para publicar con éxito. Traducido por Sylvia Podolsky Ostrowiak y Alejandra Medrano. 1 edition. FLACSO México.

8 http://exchanges.wiley.com/authors/types-of-peer-review_706.html

9 Spier, Ray. 2002. The History of the Peer-Review Process. Trends in Biotechnology 20 (8): 357-58. doi:10.1016/S01677799(02)01985-6.

10 Pulverer, B. 2011. «Transparency in Peer Review». Nature Materials 10 (2): 81-81. doi:10.1038/nmat2952.

11 «Transparent Peer Review at Nature Communications». 2015. Nature Communications 6 (diciembre): 10277. doi:10.1038/ ncomms10277.

12 Programa General de Información y UNISIST, y UNESCO. 1983. Guía para la redacción de artículos científicos destinados a la publicación. Segunda edición. París: UNESCO.

13 Wicherts, Jelte M., y Marjan Bakker. 2012. "Publish (your data) or (let the data) perish! Why not publish your data too?" Intelligence 40 (2): 73-76. doi:10.1016/j.intell.2012.01.004.

$14 \mathrm{http} / / /$ www.biomedcentral.com/submissions/editorialpolicies\#ethics+and+consent

15 Ecological Archives: Data Papers, Supplements, and Digital Appendices for ESA Journals [http://www.esapubs.org/archive/ default.htm]

16 Newman, P., y P. Corke. 2009. «Editorial: Data Papers -- Peer Reviewed Publication of High Quality Data Sets». The International Journal of Robotics Research 28 (5): 587-587. doi:10.1177/0278364909104283.

17 http://www.gbif.org/resource/80640

18 https://knb.ecoinformatics.org/\#external//emlparser/docs/index. html

19 http://rs.tdwg.org/dwcl

20 http://www.earth-system-science-data.net/about/aims_and_scope. html

21 http://www.nature.com/sdata/

22 http://zookeys.pensoft.net/

$23 \mathrm{http} / / /$ biodiversitydatajournal.com/

24 Heidorn, P. Bryan. 2008. «Shedding Light on the Dark Data in the Long Tail of Science». Library Trends 57 (2): 280-99. doi:10.1353/lib.0.0036.

25 Sanchez Upegui, Alexander Arbey. 2011. Manual de redacción académica e investigativa: cómo escribir, evaluar y publicar artículos. Medellín: Católica del Norte Fundación Universitaria.

26 Cisneros-Estupiñán, Mireya, y Giohanny Olave-Arias. 2012. Redacción y publicación de artículos científicos: enfoque discursivo. Bogotá: Ecoe Ediciones.

27 Ritter, Robert. 2002. The Oxford Guide to Style. 2nd Edition edition. Oxford ; New York: Oxford University Press.

28 Turabian, Kate L. 2007. A Manual for Writers of Research Papers, Theses, and Dissertations. 7th Edition. London: University of Chicago Press.

29 Freedman, Leonard P., Iain M. Cockburn, y Timothy S. Simcoe. 2015. "The Economics of Reproducibility in Preclinical Research". PLoS Biol 13 (6): e1002165. doi:10.1371/journal. pbio. 1002165.

30 Begley, C. Glenn, y Lee M. Ellis. 2012. "Drug Development: Raise Standards for Preclinical Cancer Research". Nature 483 (7391): 531-33. doi:10.1038/483531a.

31 Mobley, Aaron, Suzanne K. Linder, Russell Braeuer, Lee M. Ellis, y Leonard Zwelling. 2013. "A Survey on Data Reproducibility in Cancer Research Provides Insights into Our Limited Ability to Translate Findings from the Laboratory to the Clinic". PLoS ONE 8 (5): e63221. doi:10.1371/journal. pone. 0063221 .

32 Prinz, Florian, Thomas Schlange, y Khusru Asadullah. 2011. "Believe It or Not: How Much Can We Rely on Published Data on Potential Drug Targets?" Nature Reviews Drug Discovery 10 (9): 712-712. doi:10.1038/nrd3439-c1. 
33 Collaboration, Open Science. 2015. "Estimating the Reproducibility of Psychological Science". Science 349 (6251): aac4716. doi:10.1126/science.aac4716.

34 http://validation.scienceexchange.com/\#/

35 «Announcement: Reducing our irreproducibility». 2013. Nature 496 (7446): 398-398. doi:10.1038/496398a.

36 http://www.nature.com/authors/policies/checklist.pdf

37 Fang, F. C., R. G. Steen, y A. Casadevall. 2012. «Misconduct Accounts for the Majority of Retracted Scientific Publications». Proceedings of the National Academy of Sciences 109 (42): 17028-33. doi:10.1073/pnas.1212247109.

38 Steen, R. Grant, Arturo Casadevall, y Ferric C. Fang. 2013. «Why Has the Number of Scientific Retractions Increased?» PLoS ONE 8 (7): e68397. doi:10.1371/journal.pone.0068397.

39 Fanelli, Daniele. 2013. «Why Growing Retractions Are (Mostly) a Good Sign». PLoS Med 10 (12): e1001563. doi:10.1371/ journal.pmed.1001563.
40 Popper, Karl R. 1980. La logica de la investigacion cientifica. $5^{\circ}$ reimpresión. Madrid: Editorial Tecnos.

41 Lakatos, Imre. 1989. La metodología de los programas de investigación científica. Primera edición castellano. Madrid: Alianza Editorial.

$42 \mathrm{http} / /$ www.icmje.org/recommendations/browse/roles-and-responsibilities/defining-the-role-of-authors-and-contributors.html

43 Frische, Sebastian. 2012. "It is time for full disclosure of author contributions". Nature 489 (7417): 475-475. doi:10.1038/489475a.

44 Dance, Amber. 2012. “Authorship: Who's on first?” Nature 489 (7417): 591-93. doi:10.1038/nj7417-591a.

45 Acuna, Daniel E., Stefano Allesina, y Konrad P. Kording. 2012. "Future Impact: Predicting Scientific Success". Nature 489 (7415): 201-2. doi:10.1038/489201a.

46 Allen, Liz, Jo Scott, Amy Brand, Marjorie Hlava, y Micah Altman. 2014. «Publishing: Credit where credit is due». Nature 508 (7496): 312-13. doi:10.1038/508312a. 